\title{
TÉCNICAS DE INTERPOLAÇÃO PARA A PESQUISA EM CLIMATOLOGIA REGIONAL E AGROCLIMATOLOGIA
}

\author{
MURARA, Pedro - pedro.murara@uffs.edu.br \\ Universidade Federal da Fronteira Sul / UFFS
}

\begin{abstract}
RESUMO: Com o objetivo de discutir a pesquisa em Climatologia Regional e Agroclimatologia o artigo apresenta uma discussão desses conceitos com o intuito de demostrar as relações de convergência entre esses dois ramos da Climatologia Geográfica. Apoiado em um estudo de caso, apresentamos os métodos e técnicas de interpolação de dados climáticos, proporcionando ao leitor a compreensão de diferentes interpretações diante da seleção de uma das cinco técnicas: IDW, Spline, Kriging, Natural Neighbor e Topo to Raster. Os resultados evidenciaram que, para além da apresentação visual das informações, isto é, sua espacialização, a seleção de uma das técnicas demostra os objetivos que o usuário pretende enfatizar no uso de seus dados e informações. Ainda perante o desafio de apresentar enfrentamentos diante das variabilidades e alterações climáticas contemporâneas se enfatiza que estudos e pesquisas de regionalização do clima e dos seus elementos se fazem necessários e podem ser utilizados para o auxílio na caracterização de zoneamento agroclimático.
\end{abstract}

PALAVRAS-CHAVES: Precipitação; Espacialização; Mapeamento; ArcGis

INTERPOLATION TECHNIQUES FOR RESEARCH IN REGIONAL CLIMATOLOGY AND AGROCLIMATOLOGY

ABSTRACT: Aiming to discuss research in Regional Climatology and Agroclimatology, this paper presents a discussion of these concepts in order to demonstrate the relations of convergence between these two topics of Geographic Climatology. Based on a case study, the article presents methods and techniques for interpolating climatic data, with the aim of contributing to the understanding of different interpretations in the selection of one of the five techniques: IDW, Spline, Kriging, Natural Neighbor and Topo to Raster. The results evidenced that rather than visual differences in information the mapping and the selection of one of the techniques demonstrates the objectives that the user intends to emphasize in the use of his data and information. Furthermore with the challenge of presenting confrontations facing the contemporary variability and climate change, it is emphasized that studies and research on climate regionalization and its elements, are necessary and can be used to aid in the characterization of agroclimatic zoning.

KEYWORDS: Rainfall; Spatialization; Mapping; ArcGis.

TÉCNICAS DE INTERPOLACIÓN PARA LA INVESTIGACIÓN EN CLIMATOLOGÍA REGIONAL Y AGROCLIMATOLOGÍA

RESUMEN: Con el objetivo de discutir la investigación en Climatología Regional y Agroclimatología, este artículo presenta una discusión de esos conceptos con el fin de demostrar las relaciones de convergencia entre esas dos ramas de la Climatología Geográfica. En el artículo se presentan los métodos y técnicas de interpolación de datos climáticos, proporcionando al lector la comprensión de diferentes interpretaciones ante la selección de una de las cinco técnicas: IDW, Spline, Kriging, Natural Neighbor y Topo to Raster. Los resultados evidenciaron que además de la presentación visual de la información, o sea, su espacialización, la selección de una de las técnicas muestra los objetivos que el usuario pretende enfatizar en el uso de sus datos e información. Además, ante el reto de presentar enfrentamientos frente las variabilidades y cambios climáticos contemporáneos, se enfatiza que estudios e investigaciones de regionalización 
del clima y de sus elementos, se hace necesario y puede ser utilizado para la ayuda en la caracterización de zonificación agroclimática.

PALABRAS CLAVE: Lluvia; Espacialización; Mapeo; ArcGis.

\section{INTRODUÇÃO}

O presente texto está estruturado a partir da apresentação e da discussão resultantes da mesa redonda intitulada Climatologia Regional e Agroclimatologia: métodos, técnicas e enfrentamentos diante das alterações climáticas, que ocorreu na primavera de 2018, quando do XIII Simpósio Brasileiro de Climatologia Geográfica, realizado em Juiz de Fora, Minas Gerais.

A proposta que aqui segue se embasa na conceituação da Climatologia Regional e da Agroclimatologia, como intuito de estabelecer relações convergentes entre esses ramos da pesquisa em Climatologia Geográfica. Com o objetivo de apresentar os enfrentamentos diante das variabilidades e alterações climáticas contemporâneas, destacamos a importância da regionalização do clima e dos seus elementos climáticos para o auxílio na caracterização de zoneamento agroclimático. Dessa forma, selecionamos entre os métodos e as técnicas utilizadas a interpolação de dados a partir da realidade de um estudo de caso.

É importante deixar claro que, no presente texto, trataremos da Climatologia enquanto ramo do conhecimento da Ciência Geográfica. Por esse motivo, muito embora seja por vezes elencada em uma ou outra ciência, como por exemplo a Meteorologia, nosso foco será sobre a Climatologia Geográfica.

\section{CLIMATOLOGIA REGIONAL}

A Climatologia, por se tratar de um campo amplo de investigações, pode ser (e, geralmente, é) classificada a partir de subdivisões para a realização de suas pesquisas. Com base no objetivo da pesquisa enfatizada ou na escala do fenômeno atmosférico estudado, uma diversidade de campos e subdivisões são possíveis para o estudo climático, desde a sinótica, física, dinâmica, aplicada, histórica, paleoclimática, até a Climatologia Regional, entre outras.

Por região entendemos, a partir do senso comum, a identificação de uma área por sua localização e extensão. No entanto, para a Ciência Geográfica o conceito de região ganha complexidade e caráter científico, sendo discutido desde a origem dessa ciência, avançando e encontrado diferentes visões e perspectivas de análise (GOMES, 2006). Para as investigações em climatologia, o conceito de região se desenvolve principalmente sob o viés neopositivista, no qual a região é utilizada como um recorte espacial da investigação geográfica. Dessa forma, são eleitos determinados fatores (elementos climáticos ou apenas um único elemento do clima) que, uma vez analisados (utilizando-se principalmente analises estatísticas) produzirão diferentes classificações espaciais (áreas que são identificadas por meio de mapeamento), ou seja, diferentes regiões.

Nesse contexto, e baseado em Machado e Assis (2017), a Climatologia Regional é entendida a partir da descrição dos climas segundo das condições atmosféricas de uma determinada porção do espaço ou de um elemento 
climático (MENEZES et al., 2015; RAMOS et al., 2017). Portanto, os estudos da Climatologia Regional se caracterizam com o objetivo inicial de diferenciação de áreas ou, ainda, comparação entre áreas. Seja o estudo do clima ou de um de seus elementos constituintes, uma Climatologia Regional caracteriza determinada porção do espaço e possibilita ao investigador compreender desde a variabilidade espaço-climática até como a distribuição de seus elementos se organizam para uma determinada região.

Portanto, a caracterização regional do clima impõe, aos investigadores, o desafio de converter a grande massa de dados meteorológicos e climáticos disponíveis, coletados a partir de localidades especificas (pontuais), e espacializar essas informações para áreas onde não há coleta de dados, conforme nos apresenta Mendonça e Danni-Oliveira (2007). O desafio se torna ainda maior diante dos problemas que aqui se apresentam, que podem ser a ausência de pontos de coletas, as falhas de dados que resultam em rupturas nas séries temporais, bem como a mudança de localidade das estações ou, até mesmo, a sua desativação e fechamento. Portanto, para a realização de uma Climatologia Regional, os desafios apresentados são tantos, quanto o rigor técnico necessário para a realização desses estudos baseados em séries de dados homogêneas e robustas do ponto de vista estatístico (ACQUAOTTA; FRATIANNI, 2014; MURARA, et al., 2018)

Mesmo diante desses desafios, a Climatologia Regional possibilita o conhecimento das características atmosféricas e climáticas que, por sua vez, são utilizados como um fator de extrema importância para a produção do espaço. Ou seja, pode ser entendida como um estudo inicial, preliminar, diante do fato de ser necessário, primeiro, caracterizar e descrever o clima e as condições atmosféricas de uma porção do espaço para que, em um segundo momento, se possa se planejar os usos ideais e possíveis que serão executados nessa localidade, com base nas condições e variabilidades climáticas que ali são registradas. Conhecer, por exemplo, as características das precipitações pluviais, ou mesmo da variabilidade das temperaturas de uma determinada região, possibilita que o uso e as alterações efetuadas naquelas localidades sejam planejados com base na sua dinâmica atmosférica.

Muito embora os avanços tecnológicos tenham possibilitado ao ser humano o desenvolvimento de atividades e práticas sociais em ambientes diversos, algumas ações são melhor executadas quando há condições climáticas que favoreçam o seu desenvolvimento, evitando, assim, o aporte e o investimento em tecnologias que possam elevar o valor do produto final. Por exemplo, as práticas agrícolas e as atividades pecuárias possuem limitações aos diferentes condicionantes da atmosfera, isto é, embora os avanços tecnológicos possibilitem que sejam efetuadas nas diferentes zonas climáticas e faixas latitudinais, conhecer as dinâmicas e características da atmosfera na escala local e regional nos possibilita prever o desenvolvimento e as aptidões de culturas (soja, milho, feijão, arroz e etc.), sem a necessidade de grandes investimentos tecnológicos e financeiros.

Nesse contexto, a Climatologia Regional e Agroclimatologia se aproximam diante das questões de produção agrícola que envolvem o campo da subdivisão e identificação da Agroclimatologia que, por meio do uso dos estudos e investigações da Climatologia Regional, poderá avançar na identificação de áreas propícias ou com menor custo de investimento para seu desenvolvimento. 


\section{AGROCLIMATOLOGIA}

A Agroclimatologia se depara com diversas nomenclaturas de linhas de pesquisa, tais como Meteorologia Agrícola, Agrometeorologia e Climatologia Agrí-cola. Em um estudo de Wollmann \& Galvani (2013), acerca da caracterização conceitual de zoneamento climático, a Meteorologia Agrícola possui como objeto de estudo a relação entre o clima e os vegetais, cujas pesquisas estão baseadas nos processos físicos da atmosfera e dos organismos biológicos, com resultados emi $\neg$ nentemente quantitativos.

A Agrometeorologia, por sua vez, estuda a influência do clima sobre os vegetais por meio de uma perspectiva interdisciplinar, ampla e complexa, exigindo do pesquisador íntima compreensão dos problemas agrícolas e meteorológicos, considerando, ainda, as Ciências Biológicas, a Física, a Meteorologia e a Economia. Dessa forma, a Agrometeorologia tem o caráter de maximi $\neg$ zação da exploração econômica dos recursos naturais, intensificando a produção agrícola do solo, seja o seu uso voltado às atividades agropecu $\neg$ árias ou às florestais (WOLLMANN \& GALVANI, 2013).

A Climatologia Agrícola, por outro lado, avança seus estudos para demais áreas do conhecimento. Segundo Grisollet et al. (1952, apud WOLLMANN \& GALVANI, 2013), a Climatologia Agrícola contempla as escalas de análise:

1. A análise do clima como fator pedogenético;

2. O clima e as diferentes fases de produção da cultura agrícola, tais como a necessidade de irrigação e poda;

3. A influência dos elementos climáticos no desenvolvimento fenológico da cultura;

4. Os aspectos climatológicos e os mecanismos de defesa das plantas em relação às "calamidades atmosféricas", como a geada, as imposições climáticas às atividades pós-colheita da cultura, como tipo de armazenamento/conservação/ climatização e transporte;

Relação clima/altitude/relevo (relevo aqui entendido como as vertentes e sua exposição ao Sol) e suas influências ao desenvolvimento da cultura (GRISOLLET et al. 1952 apud WOLLMANN \& GALVANI, 2013).

Wollmann \& Galvani (2013) destacam que os três conceitos (Meteorologia Agrícola, Agrometeorologia e Climatologia Agrí $c$ cola) são abordados pela linha de pesquisa da Bioclimatologia Vegetal, que se preocupa com a relação clima e plantas e é colo $\neg$ cada como uma linha que procura entender as razões pelas quais determinadas espécies não vivem fora de limites de valores apresentados pe $\neg$ los diferentes atributos climáticos, ocorrendo, assim, uma distribuição geográfica e climática das plantas.

Por fim, conforme Burgos (1967), a Agroclimatologia é o ramo da Climatologia que possui como objeto de estudo o conhecimento do clima e do tempo com o objetivo de se intensificar a produção agrícola (WOLLMANN \& GALVANI, 2013). Apesar de o termo Agroclimatologia ser utilizado para o zoneamento, ou seja, a realização de um zoneamento em Agroclimatologia (agroclimático), o seu conceito pode ser interpretado à luz de Burgos (1967) como um ramo de estudo da climatologia geográfica que busca, por meio da 
compressão e do conhecimento do clima e das diferentes condições de tempo atmosférico, auxiliar na manutenção e desenvolvimento da produção agrícola.

Assim, mais uma vez cabe destacar as aproximações junto à Climatologia Regional, que pode ser entendida como aporte para os estudos em Agroclimatologia por meio do uso dos estudos e investigações que identificam áreas propícias para a produção do espaço, seja ele urbano ou, como aqui se aplica em culturas, rural.

\section{OS ESTUDOS EM CLIMATOLOGIA REGIONAL E AGROCLIMATOLOGIA}

Os estudos da Agroclimatologia estão focados na determinação de áreas climatologicamente aptas ao cultivo de culturas (FENNER, et al., 2015; FRANCISCO et al., 2016; MACHADO et al., 2017; GARCIA et al., 2018). Ou seja, os estudos agroclimatológicos, além de determinar a melhor época de semeadura de uma cultura, classificam e fragmentam espaços com características necessárias para o desenvolvimento de um determinado cultivo. Há, portanto, um enfoque no objeto de estudo, que é o produto desenvolvido (ou que venha a ser), porém, de modo não determinista da relação das condições atmosféricas para o seu desenvolvimento, ou seja, é para delimitar e identificar localidades em que o investimento com irrigação, por exemplo, possa ser minimizado em virtude das características climáticas da região.

Nesse sentido, a Agroclimatologia tem como foco a produção de uma cultura que será implantada e desenvolvida e não a prioridade do clima e as condições atmosféricas. O clima se torna um elemento que pode agregar valor e, portanto, isentar prejuízos em investimentos de produção. É evidente que qualquer alteração e variação das condições atmosféricas irá impactar positiva ou negativamente sobre a cultura, daí a importância do monitoramento e da compreensão constante das variabilidades que uma região pode apresentar.

É possível, ainda, que a questão climática, no sentido de definição do clima a partir da associação dos elementos que o caracterizam, não seja o foco principal da investigação em Agroclimatologia, de modo que se dedique a apenas um dos elementos que compõem o clima. Essa situação é provável que ocorra diante do fato de ser identificado que apenas o elemento climático em investigação é preponderante na determinação de uma cultura. Ou, ainda, no caso de a estação meteorológica possuir apenas uma série de dados consistente para aquele elemento.

Nesse sentido, o estudo da variabilidade espacial de um elemento climático se torna fundamental para o mapeamento de áreas de aptidão para agricultura, bem como para o planejamento das atividades agrícolas (FRANCISCO et al., 2016). Identificar ou analisar potencialidades agrícolas, principalmente se considerarmos a extensão territorial como condicionante para determinar épocas de semeadura mais favoráveis a uma cultura é uma atividade de pesquisa e investigação que compete aos estudiosos da Agroclimatologia.

Destacam-se nos estudos agroclimatológicos aqueles efetuados com base, principalmente, em faixas de aptidão por temperatura e deficiência hídrica, uma vez que a quantidade de água (precipitação e umidade), bem como 
a temperatura (limiar para maturação e desenvolvimento) são elementos importantes e necessários para o desenvolvimento de diferentes culturas.

Segundo Sediyama et al. (2001), faz-se necessário o conhecimento do nível de aptidão agrícola para o cultivo em uma região específica, o que exige levantamento, organização e análise de dados climáticos peculiares às necessidades de tal cultura. Pois o planejamento para ocupação racional do solo agrícola, como de qualquer outra forma de se delimitar o território ou área de interesse, demanda informações acerca das principais condicionantes do processo produtivo. Dessa maneira, o zoneamento agroclimático é desenvolvido para culturas específicas e visa identificar períodos favoráveis e desfavoráveis para o plantio (MAYER; BRAGA, 2002).

Logo, há pontos de convergência entre as pesquisas realizadas pela Climatologia Regional e Agroclimatologia. Embora a primeira esteja, principalmente, focada na compreensão da dinâmica climática em escala regional, a segunda necessita dessas informações para identificar áreas mais propícias para o desenvolvimento de sua produção agrícola. Os estudos em Climatologia Regional e em Agroclimatologia revelam, portanto, uma mescla e um elo entre suas investigações, ainda que os focos de sua aplicabilidade não sejam exatamente congruentes.

O zoneamento agroclimatológico, segundo Ometo (1981), é uma técnica utilizada para determinar regiões propícias ao desenvolvimento de determinadas culturas, sendo que as condições de ambiente, de solo e econômicas, quando favoráveis, proporcionam maior produtividade e rentabilidade. Logo, zoneamento agroclimático possibilita as delimitações microclimáticas regionais e o conhecimento de áreas com potencial para o estabelecimento de culturas agrícolas (SEDIYAMA et al., 2001; WOLLMANN; GALVANI, 2013), minimizando os riscos derivados das adversidades climáticas.

Vale ressaltar que o zoneamento agroclimático se refere à escala do macroclima, no qual as regiões identificadas podem, em última análise, ser muito gerais e pouco confiáveis, uma vez que se trata de uma escala que pode abranger valores superiores a centenas de quilômetros. Em contrapartida, as escalas microclimáticas - metros ou dezenas de quilômetros - apresentam maior detalhamento das condições de variabilidade dos elementos do clima, bem como acomodam as condições locais, por exemplo, a morfologia do relevo (topoclima) que melhor expressará as condições de uma determinada cultura (MACHADO et al., 2017).

Nesse sentido, parece-nos que o zoneamento deva ser constantemente atualizado, visando obter maiores informações sobre as condições climáticas de culturas selecionadas e, sobretudo, proporcionar maior retorno dos investimentos, a médio e longo prazo, uma vez que quaisquer que sejam as variabilidades climáticas, ou de um elemento do clima, se refletirão em mudanças no padrão de plantio e, com isso, em necessidade de investimento naquela cultura. Para tanto, há a necessidade de obtenção de dados meteorológicos mais consistentes, bem como a utilização de técnicas modernas para identificação de áreas mais propícias ao desenvolvimento das culturas (SANTOS, 1999).

Merece destaque, também, o zoneamento agrícola com vistas aos riscos climáticos, que Wollmann e Galvani (2013) consideram para além das variáveis 
analisadas (clima, solo e planta), funções matemáticas e estatísticas (frequencistas e probabilísticas), com o objetivo de quantificar o risco de perda das lavouras com base no histórico de ocorrência de eventos climáticos adversos.

A relação de eventos climáticos extremos, por meio de sua identificação para regiões e localidades propícias a sua ocorrência, possibilita ações no enfrentamento diante de períodos de estiagem, extremos de precipitação, geadas, entre outros. Nesse sentido, mais uma vez destacamos a importância da realização de regionalizações do clima e a elaboração de zoneamento climáticos com vistas à compreensão de ações que possam ser implantadas a partir da compreensão e da variabilidade de setores da atmosfera.

Com base nos estudos e pesquisa desenvolvidos nessa perspectiva, ficou evidente que a técnica de mapeamento das informações é mais utilizada, uma vez que possibilita ao interessado visualizar os setores, as áreas e as regiões que caracterizam as diferentes áreas de acordo como foco da investigação.

\section{MÉTODOS E TÉCNICAS}

Para a realização de zoneamentos climáticos e/ou identificação de regiões climáticas, são necessárias técnicas de espacialização das informações que utilizam, quase que exclusivamente, dados locais por meio de análises matemáticas e estatísticas. Atualmente, a identificação de zoneamento climático na Ciência Geográfica é operacionalizada majoritariamente em ambiente SIG e, portanto, necessita de bases de dados temáticos georreferenciados para execução de suas análises espaciais (NEVES et al., 2013).

Não podemos deixar de lembrar da utilização do método de Thornthwaite e Mather (1955) para o cálculo da evapotranspiração potencial da cultura em análise, bem como a utilização da soma térmica (soma dos graus-dia acumulados), que consiste na soma das unidades térmicas acima da temperatura base da cultura, que utilizada em conjunto com épocas de semeadura determina as melhores épocas de cultivo. No entanto, isso só é possível com base em uma robusta consistência de dados meteorológicos, que permitirá ao investigador identificar a variabilidade natural do regime pluviométrico, por exemplo, e, assim, interpolar informações para sua localização do entorno.

A interpolação de dados é o método que consiste em construir e projetar novos conjuntos de informações a partir de dados pontuais obtidos de uma amostragem consistente. Ou seja, faz-se necessário um conjunto existente de dados previamente conhecidos. Dessa forma, matematicamente e por meio da interpolação de dados, pode-se construir uma função que, aproximadamente, "se encaixa" nesses dados pontuais, conferindo-lhes, então, a continuidade desejada para localidades que não possuem tais informações.

Em uma busca por referências, é possível identificar muitos métodos de interpolação aplicáveis a diferentes propósitos. Os mais práticos adotam relações lineares, porém, produzem resultados considerados grosseiramente imprecisos. Os mais complexos, por outro lado, se encontram geralmente inseridos em programas computacionais e softwares cujos algoritmos não são 
fornecidos ou não são tão simples de serem compreensíveis para a ampla maioria dos usuários.

É nesse contexto que o presente artigo objetivou apresentar modelos de interpolação de dados meteorológicos, com o intuito de proporcionar ao leitor a compreensão de diferentes possibilidades de interpretações diante da seleção de uma das cinco técnicas selecionadas.

\section{MATERIAIS E MÉTODOS}

Incialmente, foi efetuada uma busca no site da Biblioteca Digital Brasileira de Teses e Dissertações, bem como no portal de periódicos da Coordenação de Aperfeiçoamento de Pessoal de Nível Superior - CAPES com as seguintes palavras-chave: Meteorologia Agrícola, Agrometeorologia, Climatologia Agrí cola e Agroclimatologia, a fim de visualizar quais são os termos mais utilizados frente às pesquisas nessa área do conhecimento. 0 recorte temporal para a busca considerou todo o período de informações presente nos bancos de dados, de modo que o mais antigo registro identificado foi da década de 1980 (até o ano de 2018) para ambos os bancos de dados. Para as buscas, foram consideradas as categorias: i. títulos da obra; ii. Assunto; e iii. Geral.Posteriormente, passamos à leitura de diferentes artigos, dissertações e teses para identificar quais são as técnicas de interpolação mais utilizadas, bem como quais os critérios de escolha por parte dos pesquisadores para seu uso. Nessa etapa, ficou evidente que não há maior detalhamento ou justificativa da seleção da técnica utilizada. Portanto, nosso critério para a apresentação das técnicas foi focar nas que possuem maior utilização por parte dos pesquisadores, logo, as mais conhecidas.

Utilizamos para a representação espacial, o software ArcGis, que possui no seu pacote de dados as cinco técnicas de interpolação de dados: IDW, Spline, Kriging, Natural Neighbor e Topo to Raster. Para a exemplificação das técnicas, utilizamos como referência o estudo desenvolvido em Murara et al. (2018), que investigou a distribuição das precipitações pluviais na bacia hidrográfica do Vale do Itajaí em Santa Catarina.

Com relação à interpolação, não realizamos quaisquer alterações do formato que o software apresenta como resultado final de sua análise, possibilitando identificar a interpolação tal qual o programa realiza. Por fim, não foram inseridos os atributos necessários para a representação de um mapa, uma vez que o objetivo é deixar a figura representativa da técnica o mais simples possível para que o leitor possa extrair a representação do produto tal como o software apresenta.

\section{RESULTADOS E DISCUSSÕES}

Com base na busca efetuada dos conceitos apresentados
(Agroclimatologia, Climatologia Agrícola, Meteorologia Agrícola e
Agroclimatologia), a seguir apresentamos os gráficos dos resultados:




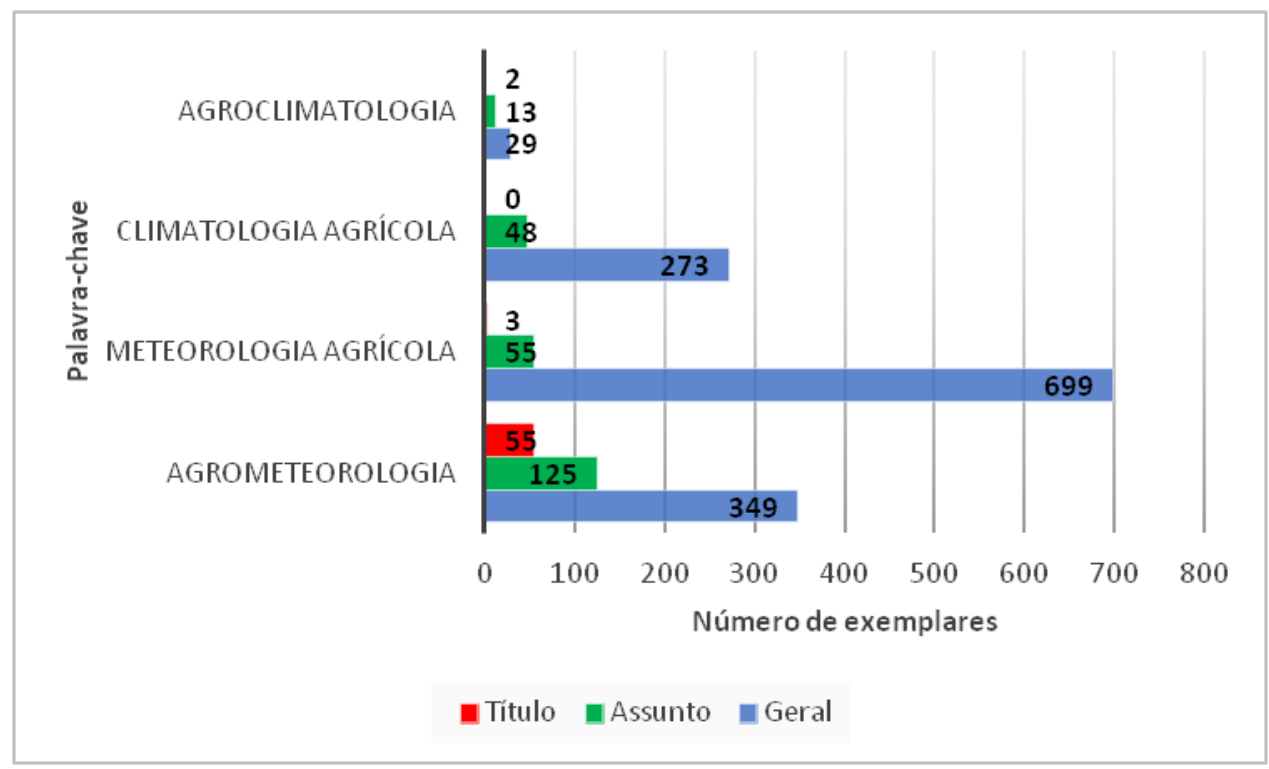

Figura 1 - Quantitativo de dissertações e teses. Fonte: BDTD (2018)

Evidencia-se, com base na Figura 1 , que o maior quantitativo de trabalhos acadêmicos efetuados pela Ciência Meteorológica é no que compete às investigações da relação das condições atmosféricas com as atividades agrícolas. Portanto, pesquisas efetuadas principalmente por meteorologistas. Os trabalhos de Agroclimatologia e Climatologia Agrícola, embora não exclusivos, tendem a ser realizados também por geógrafos.

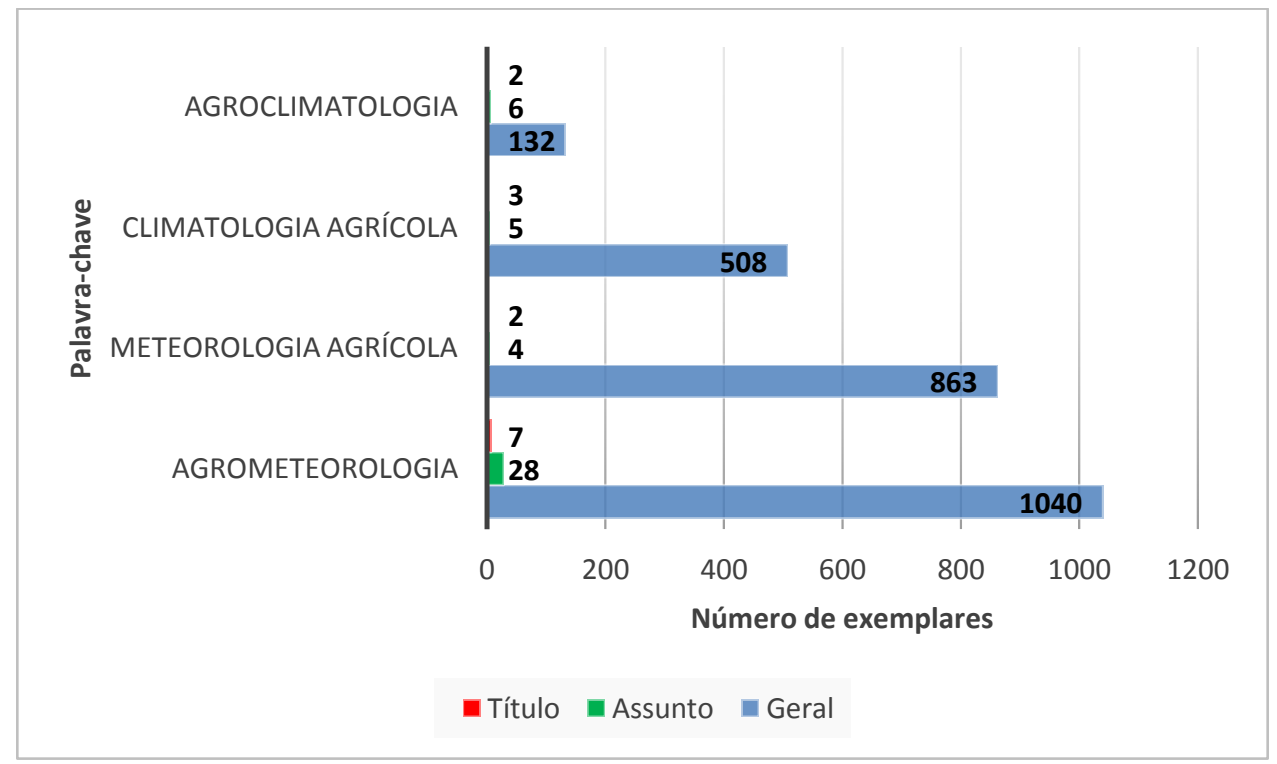

Figura 2 - Quantitativo de periódicos nacionais. Fonte: CAPES (2018)

O mesmo se verifica na Figura 2, que apresenta o quantitativo de periódicos nacionais com base nas palavras-chave de busca. Seguindo o perfil 
dos trabalhos acadêmicos defendidos (Figura 1), observamos um maior quantitativo de publicações que ocorrem na área da Meteorologia, com foco nas dinâmicas atmosféricas e sua relação com as atividades agrícolas.

Essas informações possibilitam um campo de discussão para investigações mais profícuas de quem são, de fato, os profissionais que investigam as condições e dinâmicas atmosféricas associadas a produção agrícola. Muito embora esse breve levantamento apresente indicações de que sejam principalmente realizadas por meteorologistas, faz-se ainda necessário identificar os demais profissionais, bem como entender os motivos de poucas investigações dessa natureza realizada por geógrafos. Essa afirmação se baseia na pesquisa realizada por Fialho (2010) que já apresentava, primordialmente, os estudos de climatologia com enfoque no espaço urbano como foco de investigação por parte dos geógrafos.

Das cinco técnicas aplicadas, somente a técnica do Topo to Raster (Figura 9) ultrapassa os limites dos pontos de informações e realiza o corte da espacialização baseado nas bordas do mapa de representação. As demais técnicas utilizam os limites dos pontos de informações para realizar a espacialização e o corte das informações que serão projetadas. Dessa forma, a representação terá como limite os pontos de latitude e longitudes mais extremos, podendo, como nos exemplos das figuras 3, 6, 7 e 8, não cobrir toda a sua área de representação.

Desta forma, com exceção da Figura 9 (técnica Topo to Raster), para as demais, quando da realização da interpolação de dados, são necessárias informações ou pontos de coletadas de dados para além da área de estudo, ou seja, fora dos limites da área que se pretende analisar. A alternativa utilizada para sanar essa forma de representação é por meio da utilização de informações, no exemplo de pontos pluviométricos que extrapolem a área de estudo, possibilitando, em um segundo momento, realizar um recorte sobre a área foco da pesquisa. Evidentemente, essa possibilidade é restrita ao número de estação que o entorno possui.

No exemplo utilizado no presente estudo não seria possível utilizar esse procedimento para todo o entorno, diante do fato de que a porção à direita da representação é limítrofe, em parte, ao Oceano Atlântico.

\section{IDW}

A interpolação por meio do IDW (Inverse of Distance Weighted) determina valores utilizando uma combinação linear ponderada de um conjunto de dados da amostra. Dessa forma, o peso atribuído a esse dado "criado" é uma função da distância inversa. A superfície a ser interpolada deve ser aquela de uma variável locacional dependente, logo, o IDW parte do pressuposto de que as coisas mais próximas entre si são mais parecidas do que as mais distantes. 


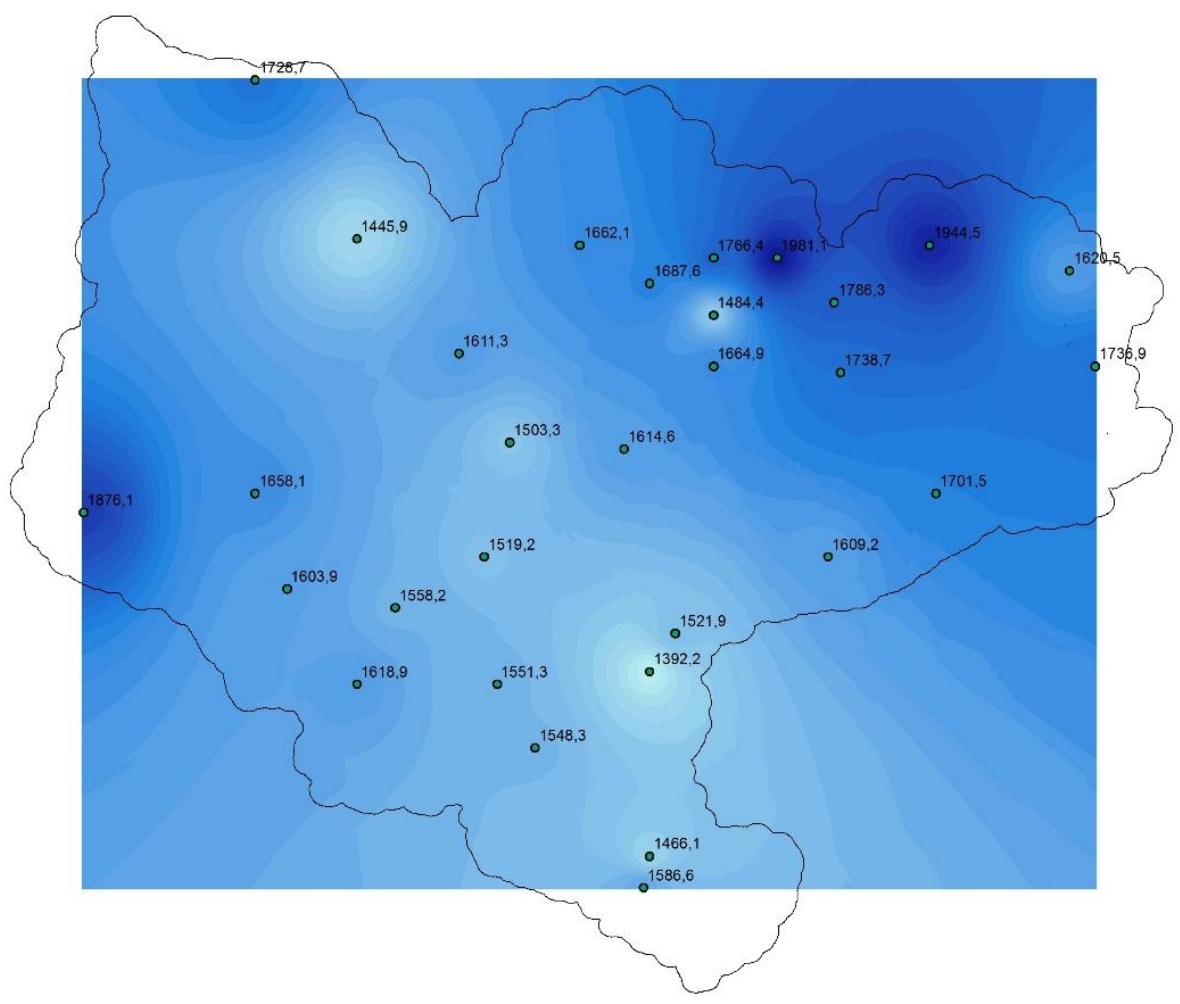

Figura 3 - Interpolação por meio do uso do IDW

A interpolação é efetuada com base na ponderação que é atribuída a pontos amostrais por meio da utilização de um coeficiente de ponderação que controlará como a influência da ponderação irá diminuir à medida que a distância a partir do ponto desconhecido aumenta. Portanto, para a espacialização de uma informação de algum local não medido, o IDW usará os valores amostrados a sua volta, que terão um maior peso do que os valores mais distantes, ou seja, cada ponto possui uma influência no novo ponto, que diminui na medida em que a distância aumenta - o que indica seu nome.

Uma vez que esse método assume que a variável que está sendo mapeada diminui em influência com a distância de sua localização amostrada, ao interpolar a distribuição das precipitações pluviais (Figura 3), os postos pluviométricos mais distantes terão menor influência porque os postos pluviométricos próximos têm mais probabilidade de retratar a realidade de uma localidade que não tem um posto pluviométrico ou sem informações.

É importante notar que o método de interpolação IDW também possui algumas desvantagens. Primeiro, a qualidade da interpolação pode diminuir se a distribuição de pontos amostrais é irregular. Além disso, os valores máximos e mínimos na superfície interpolada só podem ocorrer com base nos pontos de dados da amostra. Isso resulta frequentemente em pequenos picos e poços em torno dos pontos de dados de existentes, como mostrado na Figura 4, quando da sua espacialização em 3D. 

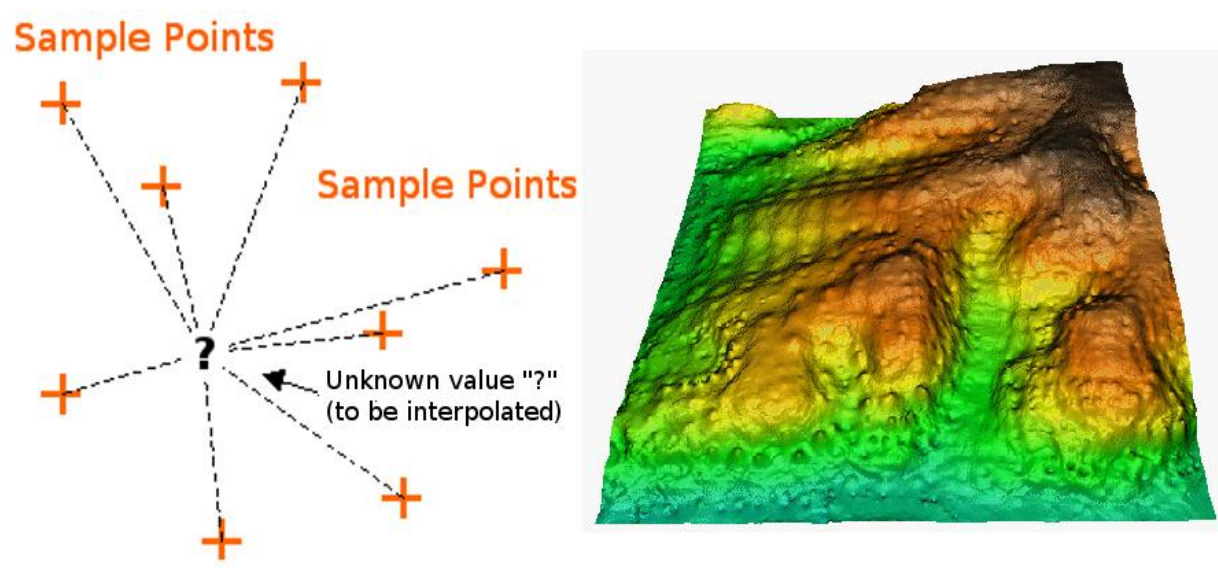

Figura 4 - A interpolação IDW é baseada na distância ponderada de um ponto amostral (à esquerda). Superfície IDW interpolada a partir de pontos vetoriais de elevação (à direita).Fonte: MITAS, L., MITASOVA, H. (1999).

\section{SPLINE}

A interpolação de dados pela técnica do Spline (Figura 6) estima valores usando uma função matemática que minimiza a curvatura total da superfície, resultando em uma superfície lisa que passa exatamente pelos pontos de entrada. Conceitualmente, um Spline é uma curva entre pontos de controle e nós (Figura 5). O modelo será mais robusto quando maior número de pontos de controle for fornecido para a espacialização da informação.

O Spline ajusta uma função matemática a um número especificado de pontos de entrada (pontos de controle) mais próximos ao passar pelos pontos de amostra (pontos de nó). Variando as posições dos pontos P1 e P2, a curva apenas varia sua inclinação, mas continua passando pelos pontos P0 e P3. Esse método é melhor para gerar superfícies com variações delicadas, como elevação, altura do lençol freático ou concentrações de poluição.

Conforme pode ser observado na Figura 6, é possível identificar, na porção central da imagem, uma tonalidade de azul escuro em destaque, como representação de um maior volume de precipitação para essa localidade. No entanto, essa informação é baseada nos dados do entorno, visto que não há qualquer estação ou ponto que a forneça. O mesmo ocorre para a porção mais inferior da imagem, na qual é possível identificar uma tonalidade de azul mais clara com indicação de diminuição das chuvas para essas localidades. É preciso destacar que essas são informações visuais que podem levar o leitor a interpretações errôneas da realidade, que por sua vez, são sanadas com o auxílio da legenda. 

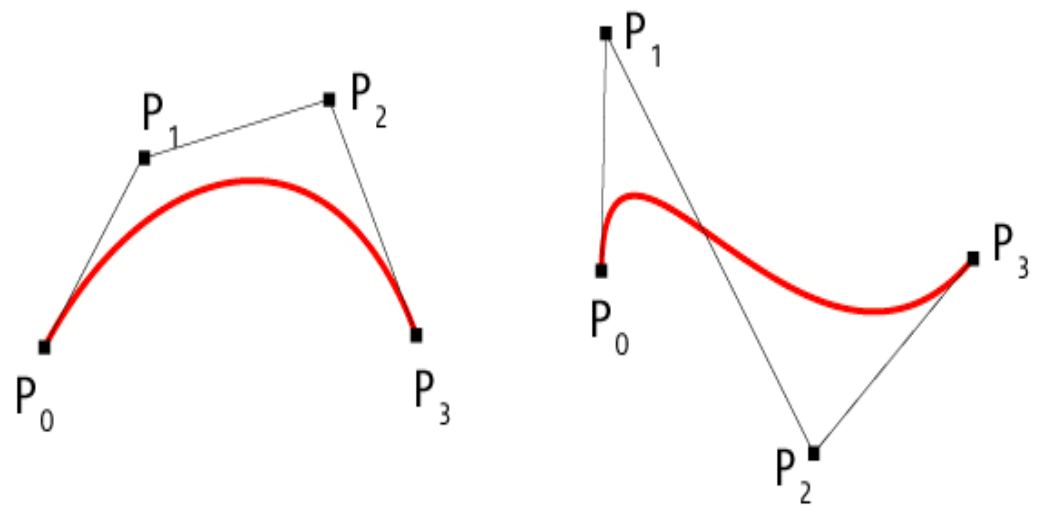

Figura 5 - Na figura, os nós são P0 e P3 e os pontos de controle são P1 e P2.

Dessa forma, a técnica de interpolação de dados por meio do uso do Spline pode ser caracterizada pela criação de informações como "ilhas" que reforçam e intensificam valores do entorno em um ponto no qual não há informações. Ou seja, embora os valores finais sempre coincidam com os dados amostrados, será possível prever mudanças mais abruptas na distribuição de dados.

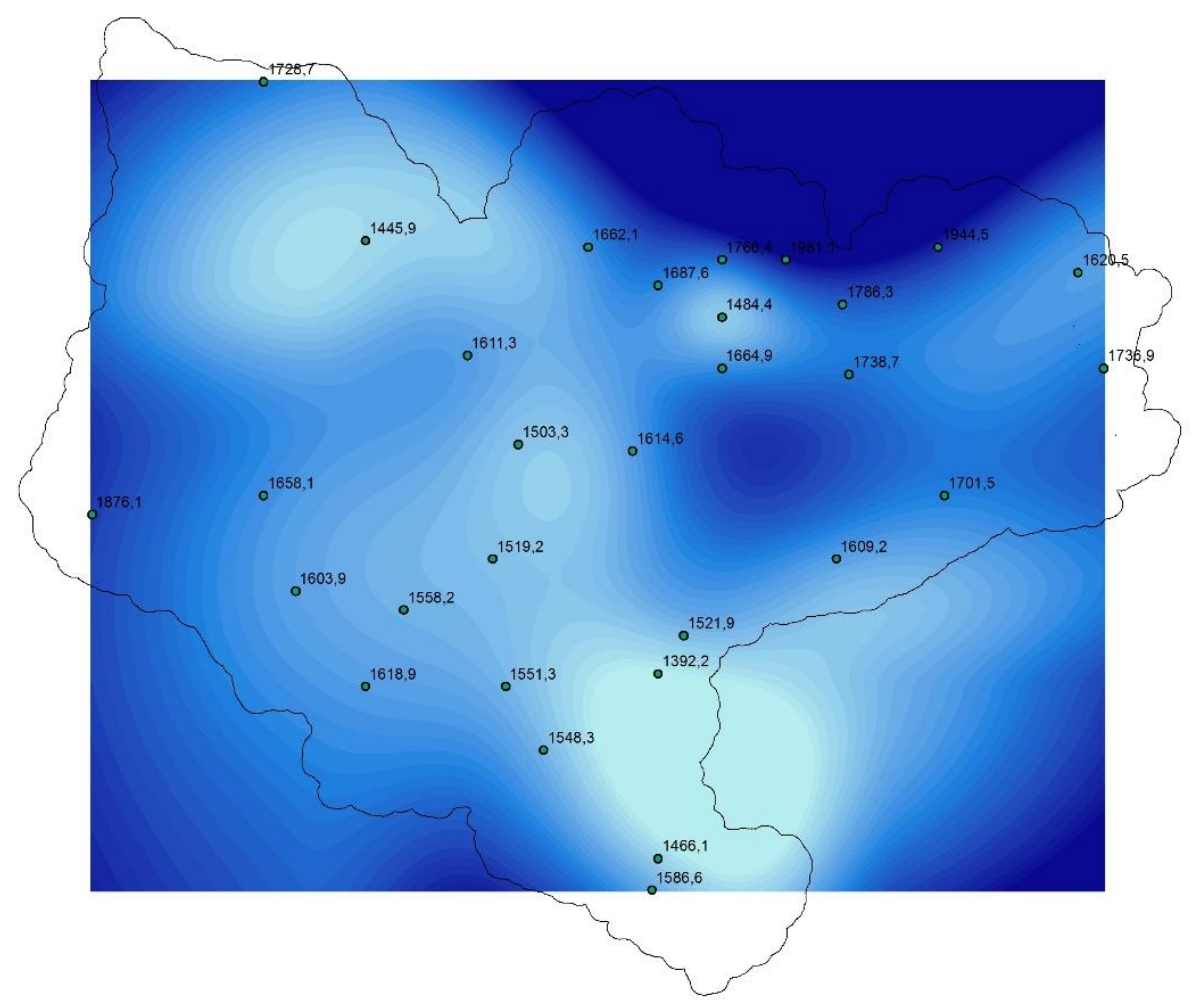

Figura 6 - Interpolação por meio do uso do Spline. 
As ferramentas de interpolação IDW e Spline são chamadas de métodos de interpolação determinística porque são diretamente baseadas nos valores medidos circundantes ou em fórmulas matemáticas especificadas que determinam a suavidade da superfície resultante.

Uma segunda família de métodos de interpolação consiste de métodos geoestatísticos que são baseados em modelos estatísticos que incluem autocorrelação, isto é, as relações estatísticas entre os pontos medidos. Por esse motivo, as técnicas geoestatísticas não apenas têm a capacidade de produzir uma superfície de previsão, mas também fornecem alguma medida da certeza ou exatidão das previsões.

\section{KRIGING}

A interpolação de dados pela técnica da Kriging, representado na Figura 7, trata-se de um procedimento geoestatístico avançado que gera uma superfície estimada a partir de um conjunto disperso de pontos com valores. Trata-se de uma possibilidade de interpolação que se baseia em uma série de técnicas de análise de regressão, sejam essas lineares ou não, que procuram minimizar a variância estimada a partir de um modelo prévio levando em consideração a dependência estocástica entre os dados distribuídos no espaço (LANDIM, 2003).

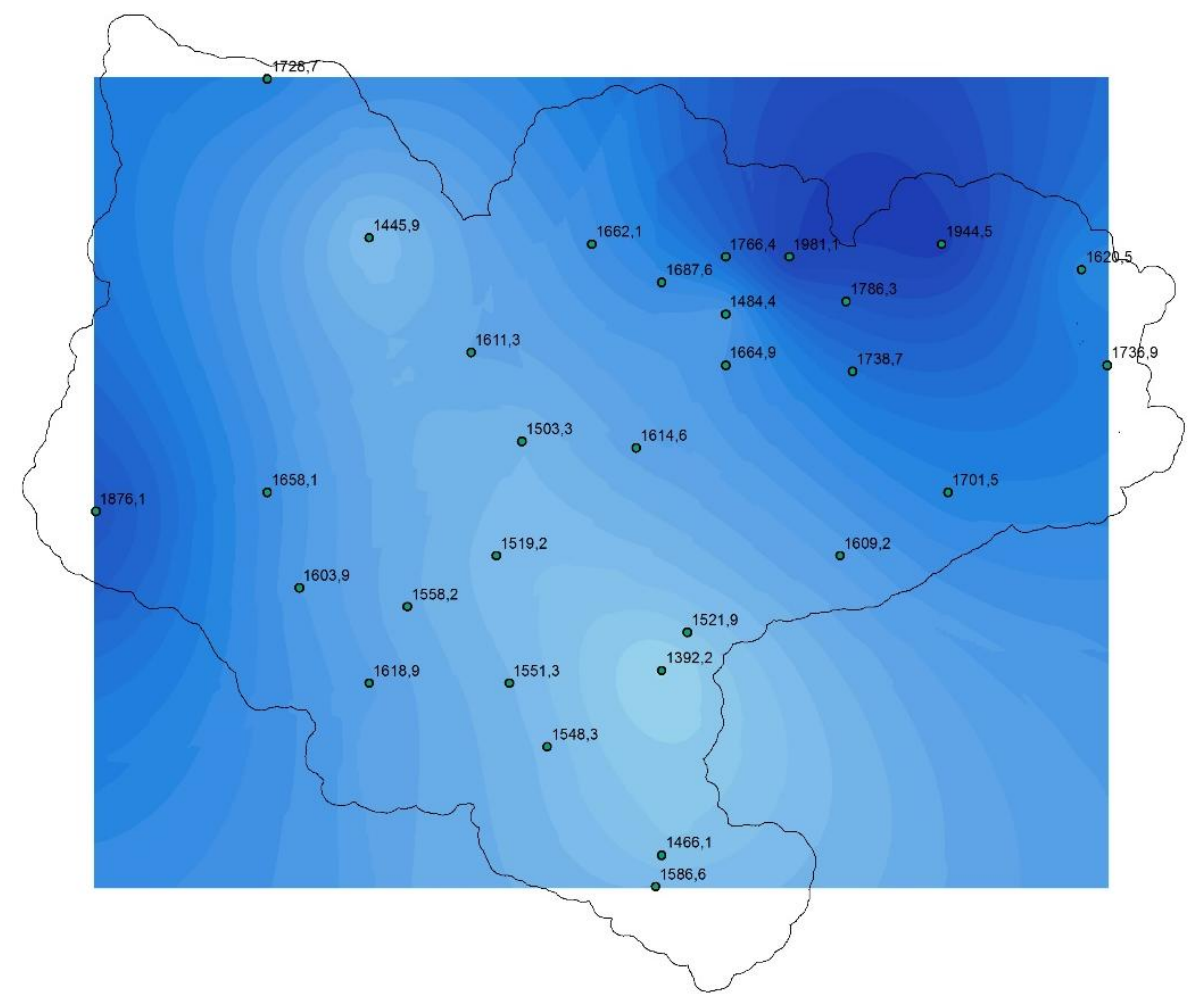

Figura 7 - Interpolação por meio do uso de Kriging. 
Desta forma, a técnica assume que a distância ou a direção entre os pontos de amostra reflete uma correlação espacial que pode ser usada para explicar a variação na superfície, ajustada em função matemática a um número especificado de pontos ou, a todos os pontos dentro de um raio especificado, para determinar o valor de saída de cada local (RAMOS, et al., 2017). Trata-se de um processo de várias etapas, incluindo análises estatísticas exploratórias dos dados, modelagem de variograma, criação da superfície e (opcionalmente) exploração de uma superfície de variância.

Essa técnica é mais apropriada quando se sabe que há uma distância espacialmente relacionada ou um desvio direcional nos dados. Trata-se do método mais utilizado para problemas ambientais uma vez que cria variações mais uniformes, não apresentando grandes variações e suavizando sua representação. Em contrapartida, esse método não permite avaliar detalhes de estruturas discretas apresentadas no revelo, por exemplo, uma vez que irá homogeneizar de modo linear as diferentes áreas internas.

\section{NATURAL NEIGHBOR}

A interpolação de dados pela técnica Natural Neighbor, representado pela Figura 8, delimita a área de espacialização da informação a partir dos pontos próximos, vizinhos. Portanto, as informações não são projetadas para além dos limites de dados fornecidos. Trata-se de uma técnica que conserva as informações, porém, apresenta o limite da necessidade de um maior número de pontos de dados para sua efetiva espacialização.

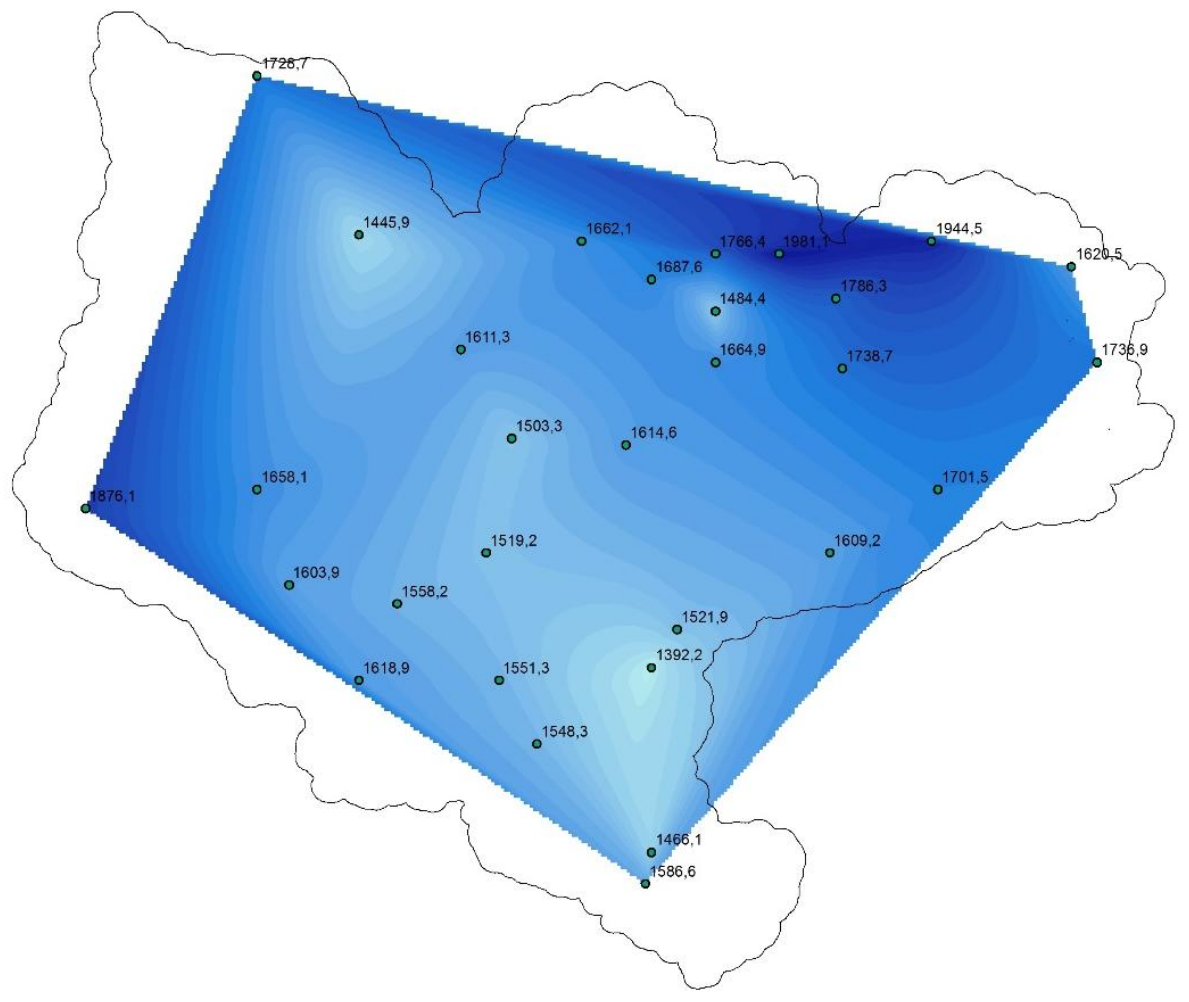

Figura 8 - Interpolação por meio do uso do Natural Neighbor. 
O algoritmo usado pela ferramenta de interpolação de vizinho natural localiza o subconjunto mais próximo de amostras de entrada em um ponto de consulta e aplica pesos a elas com base em áreas proporcionais para interpolar um valor. Também é conhecida como interpolação "roubo de área".

Suas propriedades básicas são locais, usando apenas um subconjunto de amostras que cercam um ponto de consulta, e as alturas interpoladas são garantidas dentro do intervalo das amostras usadas. Ele não infere tendências e não produzirá picos, pits, cumes ou vales que não sejam representados pelas amostras de entrada. A superfície passa pelas amostras de entrada e é lisa em todos os lugares, exceto nos locais das amostras de entrada.

\section{TOPO TO RASTER}

A interpolação de dados pela técnica do Topo to Raster (Figura 9) é um método de interpolação projetado especificamente para a criação de modelos digitais de elevação (DEMs) hidrologicamente corretos. Segundo Nogueira (2009), a técnica foi baseada nos trabalhos desenvolvidos por Hutchinson $(1998,1999)$ em seu programa ANUDEM. Os pontos positivos dessa técnica estão no fato de permitir que os resultados espaciais considerem curvas de níveis, cursos de rios e delimitações de bacias, para que possam ser utilizados durante a interpolação, diminuindo, assim, possíveis erros que possam ser representados pelo método.

O Topo to Raster interpola valores de elevação para uma imagem enquanto impõe restrições que asseguram uma estrutura de drenagem conectada e corrigir representações de cumes e fluxos de dados de contorno de entrada.

Como tal, é o único interpolador ArcGIS projetado especificamente para trabalhar de forma inteligente com entradas de contorno. Essa técnica interpola, por exemplo, dados de clima em uma grade regular, de modo interativo, gerando grades sucessivamente menores, minimizando a soma de uma de penalização de rugosidade e a soma dos quadrados dos resíduos. 


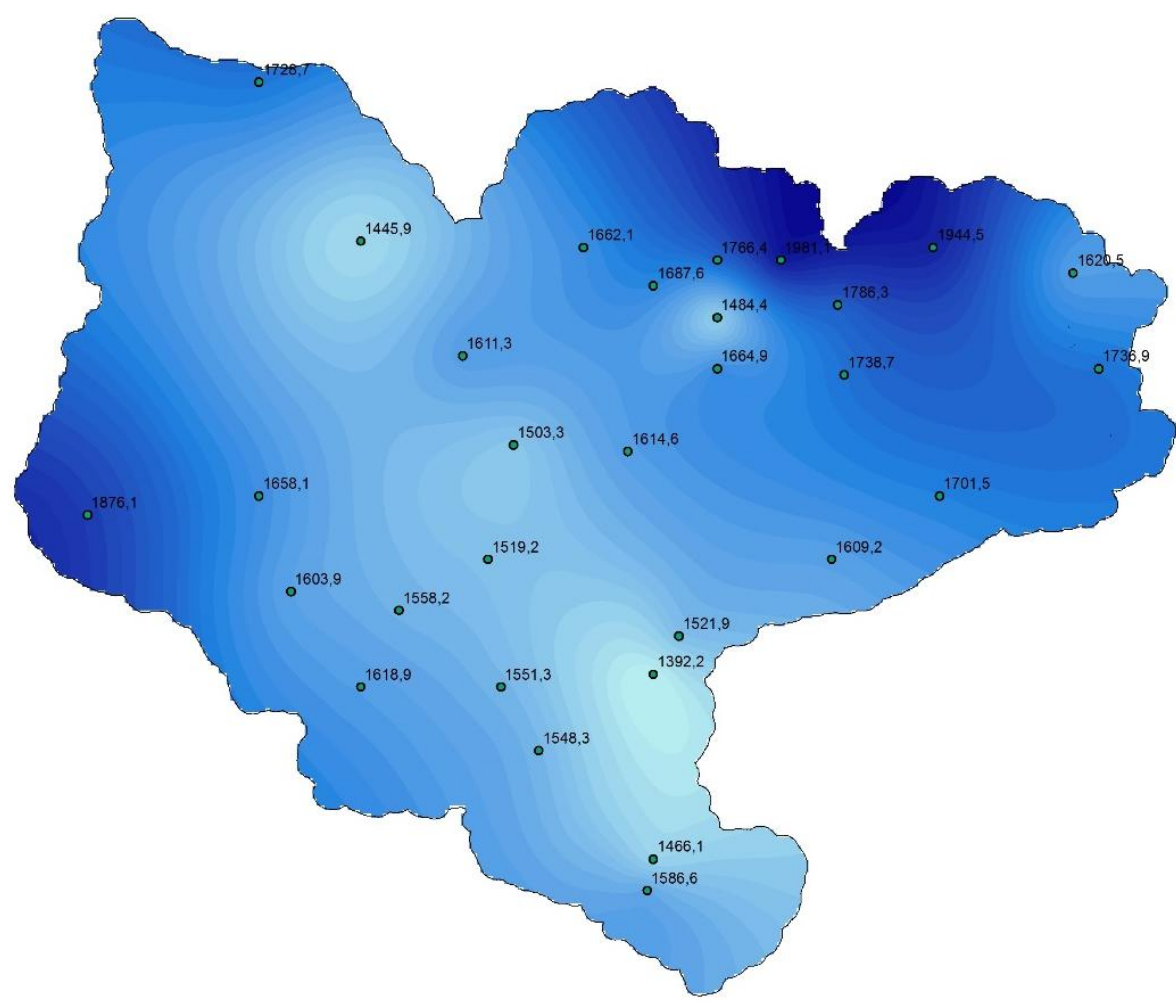

Figura 9 - Interpolação por meio do uso do Topo to Raster.

\section{CONCLUSÕES}

Conforme observações realizadas na revisão bibliográfica, as investigações por meio da compreensão das dinâmicas atmosféricas em associação com as atividades de desenvolvimento agrícola, embora sejam desenvolvidas por geógrafos estudiosos do clima, apontam para maior participação de investigações efetuadas por meteorologistas.

Metodologicamente, embora as abordagens em Climatologia Regional e Agroclimatologia são diversas, porém, ficou evidente que a primeira, pode servir de subsídio para os estudos da segunda. Portanto, conclui-se que ambas se complementam e fazem uso, principalmente, da técnica de mapeamento para a espacialização de seus dados e informações.

Salvas as problemáticas que envolvem as análises de dados e sua consistência e robustez, a realização de métodos de interpolação permite criar modelos digitais de precisão e variações entre as técnicas utilizadas. Os resultados obtidos permitem observar a diferença no desempenho dos métodos de interpolação que reagem de maneira diferente, conforme apresentados quando utilizados a partir da mesma fonte de dados. Os resultados, portanto, demonstram que as diferentes interpolações apresentam desempenho muito diferente um do outro e, por isso, fazem-se necessários diferentes testes antes de se selecionar um ou outro desses métodos, especialmente se as áreas analisadas têm variações topográficas significativas (variações altimétricas). 
Evidencia-se que a seleção do método de interpolação é uma importante etapa, pois a precisão e a acurácia de cada representação espacial dependerão da sua escolha que deverá estar em consonância com o fenômeno a ser representado. Conforme apresentado, o método Topo to Raster inicialmente diferencia-se das demais por apresentar como corte na espacialização dos dados de entrada o limite das bordas do polígono que será representado. As demais técnicas - IDW, Spline, Kriging e Natural Neighbor - apresentam como limite de espacialização os pontos de informações de dados inseridos no software, sendo necessário para esses casos, o usuário inserir informações para além da área objeto de estudo ou, estender o resultado encontrado nas bordas do polígono para além da informação pontual coletada.

O IDW é recomendável para situações em que a malha de dados e informações possui uma distribuição equivalente e equidistante com relação ao espaço em que será analisado, uma vez que toma como cálculo para a representação a proximidade dos pontos. Portanto, em análise de espacialização de dados climáticos, o IDW deverá ser utilizado quando a área de estudo possui uma distribuição de pontos de coleta suficiente e abrangente de toda a realidade a ser projetada.

O mesmo procedimento é utilizado para o Spline, no entanto, este irá reforçar e intensificar os valores entorno do ponto de informações. Ou seja, diferencia-se do IDW uma vez que enfatiza mudanças abruptas na distribuição de dados, representando no mapeamento os pontos que podem ser identificados como tendenciosos uma vez que sua técnica de curvaturas reforça a distribuição dos pontos de coleta de informações que foram inseridos. Como resultado, identificam-se picos e formações de "ilhas".

A Kriging sem dúvida é o método mais utilizado para a espacialização de dados climáticos, uma vez que cria variações mais uniformes, não apresentando grandes variações e suavizando sua representação já que homogeneíza as informações apresentadas.

A utilização da técnica de interpolação via Natural Neighbor é aconselhável quando há um maior número de pontos de dados para a espacialização das informações. Uma vez que este método utiliza os vizinhos mais próximos e utiliza-se desta informação para interpolar os dados, desconsidera as feições do relevo, por exemplo, que no caso da climatologia resultam em variações espaciais dos seus elementos.

Desta forma, entre as aplicações apresentadas e diante do fato concreto que os estudiosos da climatologia se deparam com o reduzido número de estações meteorológicas a Kriging representa o produto final com melhor desempenho diante dos fatos apresentados. Sem dúvida a interpolação pelo método Natural Neighbor merece destaque, porém, deverá ser utilizada quando houver uma malha de pontos coleta de dados que cubra de maneira homogenia a área de estudo. No entanto, o Topo to Raster é o único que considerem curvas de níveis, cursos de rios e delimitações de bacias, melhor representando desta forma espacialização dos dados climáticos.

Cabe destacar que, todos os métodos apresentados estão sujeitos a ajustes do próprio software utilizado. A seleção da Kriging, Natural Neighbor ou Topo to Raster, possibilita que uma vez selecionado o método de interpolação o 
usuário possa alterar as informações, suavizando-as ou intensificando-as a partir dos mecanismos próprios de cada método.

Finalmente, os estudos em Climatologia Regional foram apresentados a partir de uma perspectiva de compreensão da dinâmica climática de uma região, que possibilita interpretar efeitos das variabilidades, mudanças e alterações climáticas, bem como possibilidade de assessoramento na elaboração de zoneamentos climáticos com foco nos indicadores de suscetibilidade agropecuários.

De modo indireto, reforça-se a necessidade de implantação de novos pontos de coleta de dados e informações, observações sistemáticas dos elementos do clima e, principalmente, seu constate monitoramento.

\section{AGRADECIMENTOS}

Aos Organizadores do XIII Simpósio Brasileiro de Climatologia Geográfica pelo convite e possibilidade de participação no debate da mesa sob a temática da Climatologia Regional e Agroclimatologia, bem como à Associação Brasileira de Climatologia pela oportunidade de publicitação deste material que resulta dos debates realizados naquela ocasião.

\section{REFERÊNCIAS}

ACQUAOTTA, F.; FRATIANNI, S. The importance of the quality and reliability of the historical time Series for the study of climate change. Revista Brasileira de Climatologia. Ano 10 - Vol. 14 - Jan-Jul, 2014.

AYOADE, J.O. Introdução à Climatologia para os Trópicos. $14^{a}$ ed. - Rio de Janeiro: Bertrand Brasil, 2010.

BARBOSA, J.P.M. Utilização de método de interpolação para análise e espacialização de dados climáticos: o SIG como ferramenta. Caminhos de Geografia. 9 (17), p. 85-96, fev-2006.

BDTD. Biblioteca Digital Brasileira de Teses e Dissertações. Disponível em: <http://www.bdtd.ibict.br/vufind/>. Acesso em: 29 out. 2018.

BURGOS, J. J. El Clima. In: BURGOS, J. J. Las Cien $\neg$ cias Agrícolas en América Latina: Progresso y Futuro. San José: Trejos Hnos, Cap. 3. p. 85-109, 1967.

CAPES. Coordenação de Aperfeiçoamento de Pessoal de Nível Superior. Disponível em: <http://www.capes.gov.br>. Acesso em: 28 out. 2018.

DROUIN, A.; SAINT-LAURENT, D. Comparaison des méthodes d'interpolation pour l'élaboration de modèles numériques d'élévation de haute précision dans la représentation micro-topographique des plaines inondables. Hydrological Sciences Journal - Journal des Sciences Hydrologiques, 55 (4), 2010.

FENNER, W.; DALLACORT, R.; MOREIRA, P.S.P.; QUEIROZ, T.M.; FERREIRA, F.S.; BENTO, T.S.; CARVALHO, M.A.C. Índice de satisfação de necessidade de água para o milho segundo safra em Mato Grosso. Revista Brasileira de Climatologia. Ano 10 - Vol. 15 - Jul-Dez, 2014. 
FIALHO, E.S. A pesquisa climatológica realizada por geógrafos brasileiros. Revista Brasileira de Climatologia. Ano 6 - Vol. 6 - Junho/2010.

FRANCISCO, P.R.N.; BANDEIRA, M.M.; SANTOS, D.; PEREIRA, F.C.; GONÇALVES, J.L.G. Aptidão climática da cultura do feijão comum (Phaseolus vulgaris) para o estado da Paraíba. Revista Brasileira de Climatologia. Ano 12 Vol. 19 - Jul-Dez, 2016.

GARCIA, S.R.; SANTOS, D.F.; MARTINS, F.B.; TORRES, R.R. Aspectos climatológicos ao cultivo da oliveira (Olea Europaea L.) em Minas Gerais. Revista Brasileira de Climatologia. Ano 14 - Vol. 22 - Jan-Jun, 2018.

GOMES, P.C.C.; O conceito de região e sua discussão. In: CASTRO, I.E.; GOMES, P.C.C.; CORRÊA, R.L. (Org.) Geografia: conceitos e temas. 9aed. - Rio de Janeiro; Bertrand, 2006.

HUTCHINSON, M.F. Calculation of hydrologically sound digital elevation models. Paper presented at Third International Symposium on Spatial Data Handling at Sydney, Australia, 1988.

HUTCHINSON, M.F. A new procedure for gridding elevation and stream line data with automatic removal of spurious pits. Journal of Hydrology. n. 106: p. 211232, 1989.

LANDIM, P.M.B. Análise estatística de dados geológicos. São Paulo: Editora UNESP, 2003. 2. ed. Rev. e ampl. 253 p.

LUPPI, A.S.L.; SANTOS, A.R.; EUGÊNIO, F.C.; BRAGANÇA, R.; PELUZIO, R.L.; SILVA, R.G. Metodologia para classificação de zoneamento agroclimatológico. Revista Brasileira de Climatologia. Ano 10 - Vol. 15 - Jul-Dez, 2014.

MACHADO, L.A.; ASSIS, W.L. Proposta metodológica de unidades topoclimáticas. Revista Brasileira de Climatologia. Ano 13 - Vol. 20 - Jan-Jul, 2017.

MACHADO, T.S.; NEVES, S.M.A.S.; SEABRA JÚNIOR, S.; NEVES, R.J. Zoneamento agroclimático do melão na região sudoeste de Mato Grosso. Revista Brasileira de Climatologia. Ano 13 - Vol. 20 - Jan-Jul, 2017.

MENDONÇA, F.; DANNI-OLIVEIRA, I.M. Climatologia: noções básicas e climas do Brasil. São Paulo: Oficina de Textos, 2007.

MENEZES, F.P.; FERNANDES, L.L.; ROCHA, E.J.P. O uso da estatística para regionalização da precipitação no estado do Pará, Brasil. Revista Brasileira de Climatologia. Ano 11 - Vol. 16 - Jan-Jul, 2015.

NEVES, G.L.; VIRGENS FILHO, J.S.; LEITE, M.L.; SANTOS, E.N. Trend of air temperature in the state os Paraná, Brazil. Revista Brasileira de Climatologia. Ano 12 - Vol. 18 - Jan-Jul, 2016.

NOGUEIRA, J.D.L. Comparação entre os métodos de interpolação (Krigagem e Topo to Raster) na elaboração da batimetria na área da folha Touros - RN. In: Anais XIV Simpósio Brasileiro de Sensoriamento Remoto, Natal, Brasil, INPE, p. 4117-4123, 25-30, abril, 2009.

MITAS, L., MITASOVA, H. Spatial Interpolation. In: P.Longley, M.F. Goodchild, D.J. Maguire, D.W.Rhind (Eds.), Geographical Information Systems: Principles, Techniques, Management and Applications, Wiley. 1999. 
MURARA, P.G.; ACQUAOTTA, F.; GARZENA, D.; FRATIANNI, S. Daily precipitation extremes and their variation in the Itajaí River Basin, Brazil. Meteorology and Atmospheric Physic, v. 130, p.1-12, 2018.

QGIS. Quantum Geographic Information System. Disponível em: https://docs.qgis.org/2.14/pt_BR/docs/user_manual/index.html. Acesso em: 01 out. 2018.

RAMOS, H.C.; DALLACORT, R.; NEVES, S.M.A.S.; DALCHIAVON, F.C.; SANTI, A.; VIEIRA, F.F. Precipitação e temperatura do ar para o estado de Mato Grosso utilizando krigagem ordinária. Revista Brasileira de Climatologia. Ano 13 - Vol. 20 - Jan-Jul, 2017.

SANTOS, A. R. Zoneamento Agroclimatológico para a Cultura do Café Conilon (Coffeacanephora L.) e arábica (Coffeaarabica L.) na Bacia do Rio Itapemirim, ES. 1999.125 f. Dissertação (Mestrado em Engenharia Agrícola) - Programa de Pós-Graduação em Engenharia Agrícola, Universidade Federal de Viçosa, 1999.

SANTOS, D. F. dos; MARTINS, F.B.; TORRES, R.R. Impacts of climate projections on water balance and implications on olive crop in Minas Gerais. Revista Brasileira de Engenharia Agrícola e Ambiental, v. 21, n. 2, p. 77-82, 2017b.

SEDIYAMA, G.C. et al. Zoneamento agroclimático do cafeeiro (Coffea arabica L.) para o estado de Minas Gerais. Revista Brasileira de Agrometeorologia, Passo Fundo, v.9, n.3: 501-509, 2001.

THORNTHWAITE, C.W.; MATHER, J.R. The water balance. Publications in climatology. Laboratory of Climatology, New Gersey, v.8, p. 104, 1955.

WOLLMANN, C. A.; GALVANI, E. Zoneamento Agroclimático: linhas de pesquisa e caracterização teórica-conceitual. Sociedade e Natureza, Uberlândia, v.25, n.1, p. 179-190, 2013. 